\title{
Recent improvements to the Cepheid distance scale
}

\author{
Wendy L. Freedman ${ }^{1}$ and Barry F. Madore ${ }^{2}$ \\ ${ }^{1}$ Carnegie Observatories \\ 813 Santa Barbara Street, Pasadena, California 91101 \\ ${ }^{2} N A S A / I P A C$ Extragalactic Database \\ IPAC/JPL/Caltech MC 100-22, Pasadena, California 91125
}

\begin{abstract}
In the course of the last decade significant advances have been made in the observations of Cepheid variables and in their successful application to the extragalactic distance scale. Much of this progress has come about as a result of new CCD and near-infrared photometry. These recent improvements are discussed, and a comparison is given of Population I Cepheids and Population II distances. The correspondence is good, with the zero points agreeing at a level of better than $15 \%$ in distance. At this same level of significance, a systematic difference between these distances scales may exist, in the sense that the RR Lyrae distances appear to be smaller than the Cepheid distances (if it is assumed, as has generally been done for extragalactic studies of RR Lyraes, that $M_{V}(R R)=0.77 \mathrm{mag}$, independent of $[\mathrm{Fe} / \mathrm{H}]$ ). However, several recentlypublished calibrations of $M_{V}(R R)$ significantly reduce this discrepancy. Finally, new Cepheid data for the nearby galaxy M81 are presented based on recent Hubble Space Telescope observations.
\end{abstract}

\section{Introduction}

Recent refinements to the extragalactic distance scale pale in comparison to the actual discovery of the statistical relation between period and luminosity for Cepheid variables. In the 1920 's, refining the Cepheid PL zero point was important, but it was a minor detail in comparison to defining what the basic scale size of the Universe was.

A significant advance came when Baade discovered the (factor of two) distinction between the brightness of Classical Cepheids (which were being observed in external galaxies) and their fainter Population II counterparts, the W Virginis stars (which were acting as the calibrators in our Galaxy). In the 1950's, Arp, Sandage, Irwin, Kraft and others (see Fernie 1969 for a comprehensive historical review) began investigating Cepheids in Galactic clusters as a means of independently setting the Cepheid zero point, this time using Population I main sequence fitting. As photometric errors were decreased, the observed statistical relations among the apparent properties of the Cepheid samples (built up from observations of Cepheids in our Galaxy, the 
Large and Small Magellanic Clouds and several other more distant members of the Local Group) were found to be systematically different in many of their properties; for example, (1) The number distributions of amplitude over period were found to be dramatically different. (2) The observed colors at fixed period were observed to differ from galaxy to galaxy. (3) The period-luminosity relations were all found to have a measurably large and what was shown to be intrinsic scatter. And, (4) some extragalactic samples even suggested that the assumption of a universal slope to the Cepheid PL relation might be erroneous. Some of these observed properties $(1,2,3)$ have stood the test of time, whereas photometric scale errors were largely responsible for (4). And while (1) and (3) appear to be reflecting intrinsic properties of the Cepheids themselves, differing amounts of internal extinction affecting the extragalactic Cepheid samples appear to be responsible for most of property (2). In some cases in fact, the extinction corrections are so large as to rival the factors of two found so long ago by Baade.

A recent review of the Cepheid distance scale has been presented in Madore and Freedman (1991), and the details will not be repeated here. In that review, the basic physics underlying the Cepheid period-luminosity (PL) and period-luminosity-color (PLC) relations was discussed; observational issues such as the effects of reddening, metallicity and photometric errors were presented in the context of the Cepheid distance scale; the distances to individual galaxies were tabulated; and finally a consistent set of data was used to provide PL relations at 7 wavelengths: $B V R I J H K$. Other recent reviews of the Cepheid distance scale include those of Madore (1986), Feast and Walker (1987), Freedman (1988a), and Jacoby et al. (1992).

The most serious systematic error embedded in the Cepheid distance scale concerned the assumption that the Cepheids in external galaxies suffered no absorption internal to the parent galaxies in which the Cepheids were being studied. This assumption was eventually shown to be false, largely as a result of the availability of new detectors over the past decade (CCDs, IR photometers and arrays). Identifying the problem and finding a solution has been one of the many important aspects of extragalactic Cepheid research undertaken in the last decade.

\section{The Significance of Recent Changes Due to New Detector Technology}

The opinion that advancements in detector technology have significantly improved the precision of the Cepheid distance scale is not universally shared however. Specifically, Tammann (1992) states that "despite the considerable progress in Cepheid research, concerning for instance the use of infrared magnitudes (which address the problem of intrinsic absorption and metallicity differences) the resulting distances have changed very little between 1974 and 1991 ([his] Table 1). On the whole the galaxies with known Cepheids have witnessed a distance increase by only 0.05 mag (2.5 percent!)". In another context, Sandage et al. (1992) reiterate the similarity of the same two calibrations. The first two columns of our Table 1 (below) list the Sandage and Tammann (1974) [hereafter ST 1974] and the Madore and Freedman (1991) [MF 1991] calibrations as tabulated by Tammann (1992). 
The agreement between the ST 1974 and the MF 1991 calibrations is certainly remarkable; and we remark upon it below, because it is almost entirely fortuitous, and as such, it is extremely misleading. Furthermore, while the question repeatedly arises concerning the differences between the Sandage and Tammann calibrations and the more modern determinations, the answers are often not known to those other than distance scale afficionados.

First, as extensively documented in Freedman (1988b), Freedman, Wilson and Madore (1990), Christian and Schommer (1987), Capaccioli et al. (1992) there are enormous scale errors in the photographic photometry on which the ST 1974 calibration was based. At $B=22 \mathrm{mag}$ such errors typically reach about $0.5 \mathrm{mag}$.

Table 1. Distances to Local Calibrators

$\begin{array}{cccc}\text { Galaxy } & \mu_{S T 1974} & \mu_{0, M F 1991} & \mu_{S T 1984} \\ & & & \\ \text { LMC } & 18.59 & 18.50 & 18.95 \\ \text { SMC } & 19.27 & 18.87 & - \\ \text { NGC 6822 } & 23.95 & 23.59 & - \\ \text { IC 1613 } & 24.43 & 24.42 & - \\ \text { M31 } & 24.12 & 24.44 & 24.19 \\ \text { M33 } & 24.56 & 24.63 & \mathbf{2 5 . 2 3} \\ \text { NGC 2403 } & 27.56 & 27.51 & 27.66 \\ \text { M81 } & 27.56 & 27.59 & \mathbf{2 8 . 7 3} \\ \text { M101 } & 29.3 & 29.38 & -\end{array}$

Second, the ST 1974 calibration is based almost exclusively on apparent $B$ band photometry (which is all that was readily available before the advent of red-sensitive CCDs and IR detectors). Consequently the ST 1974 calibration provides apparent (not true or internal-reddening-corrected) moduli; they have been corrected only for modest amounts of foreground absorption. As clearly shown in numerous studies over the past decade (e.g., Freedman 1986, 1988a,b; Freedman, Wilson, and Madore 1991; Madore 1985; Freedman and Madore 1991) the apparent distance moduli, when measured at different wavelengths systematically decrease in a manner that is well fit by a standard interstellar extinction law. It is necessary to correct any apparent modulus for the effects of total (foreground plus internal) extinction; if the extinction is appreciable (as it is for many late-type spirals), then neglecting this term always leads to systematic overestimates of the distance.

A few examples will show the significance of these effects: (a) In Baade and Swope's Field III in M31 the apparent (CCD) $B$ modulus is 25.36 mag (Freedman and Madore 1991). The true distance modulus, based on $B V R I$ photometry is 24.44 mag, as entered in Table 1, Column 2, in such apparently good agreement with ST 1974. The $B V R I$ CCD apparent moduli are shown in Figure 1 as a function of inverse wavelength. The $B$ band apparent modulus is the relevant modulus to compare 
directly with the ST 1974 calibration which is also based on $B$ (photographic) photometry. The apparent $B$ moduli differ by 1.24 mag (!). (b) The apparent $B$ band CCD modulus of M33 is 25.04 mag (Freedman, Wilson and Madore 1991). The two $B$ moduli for M33 differ by 0.48 mag.

Third, the distances presented by MF 1991 are all computed relative to an LMC modulus of 18.50 magnitudes. On the other hand, the LMC modulus in the ST 1974 calibration is $\mathbf{1 8 . 5 9}$ mag. Comparing the two Cepheid calibrations at least requires the zero points to be consistent, while neglecting it introduces a small (but again systematic) difference in zero point of $0.14 \mathrm{mag}$ (as opposed to the $0.05 \mathrm{mag}$ offset quoted by Tammann 1991 above).

In later studies Sandage (1983) and then Sandage and Carlson (1983a) based their zero point for their distance modulus to M33 on an apparent blue LMC modulus of 18.95 mag. This LMC modulus leads to an apparent blue modulus for M33 of 25.35 mag. [That is, Sandage and Carlson (1983 - their Figure 3) found a difference in apparent $B$ moduli between M33 and the LMC of $6.4 \mathrm{mag}$. The value of 25.35 (= $18.95+6.4)$ thus includes a component due to the known LMC foreground reddening.] However, incorporating the increased distances, Sandage and Tammann (1984) tabulated their then current values for the local calibrator distances useful for the Tully-Fisher relation. These distances are given in Column 4 of Table 1 . In that compilation the adopted zero point again corresponds to the apparent $B$ modulus to the LMC of 18.95 mag. Yet these apparent moduli are equated with the true moduli in the application of the Tully-Fisher calibration. And they are increased by almost half a magnitude due purely to LMC foreground extinction, and ignore any contribution from extinction internal to either the LMC or M33.

M81 presents a unique case. The ST 1974 calibration lists NGC 2403 and M81 as having similar distance moduli of 27.6 mag. Given that only 2 Cepheids in M81 were known to Sandage, he did not attempt to derive a direct Cepheid distance to M81; rather the galaxies were assumed to be at the same distance on the basis of group membership as defined by Holmberg. Then, much later, Sandage (1984 ; i.e., in the interval between 1974 and 1992) noted that the brightest long-period Cepheids in M81 (for which he did have periods) are fainter than the brightest long-period Cepheids in NGC 2403; on the basis of this comparison he placed M81 at a distance modulus of $28.8 \mathrm{mag}$, over a full magnitude more distant than NGC 2403. (Given the existence of the PL relation however, the higher luminosities of the longest-period Cepheids in NGC 2403 are of course to be expected since they have periods of 67 days, more than a factor of two larger than those in M81 which have periods of only 30 days). Subsequent $I$-band photometry of the two known (30 day) Cepheids in M81 showed them to have magnitudes very similar to their 30-day counterparts in NGC 2403, and thereby ruled out the larger distance for M81.

The truly significant changes that did occur in the distances to many important calibrating galaxies in the period between 1974 and 1992 led at least some authors at that time (e.g. Aaronson 1985) to conclude that any value of the Hubble constant between 50 and $100 \mathrm{~km} / \mathrm{sec} / \mathrm{Mpc}$ could be obtained depending on the specific choice 
of calibrators. Fortunately, the recent convergence of the distances to nearby galaxies based on new Cepheid, RR Lyrae and other indicators, (discussed below) has largely removed this freedom of choice.

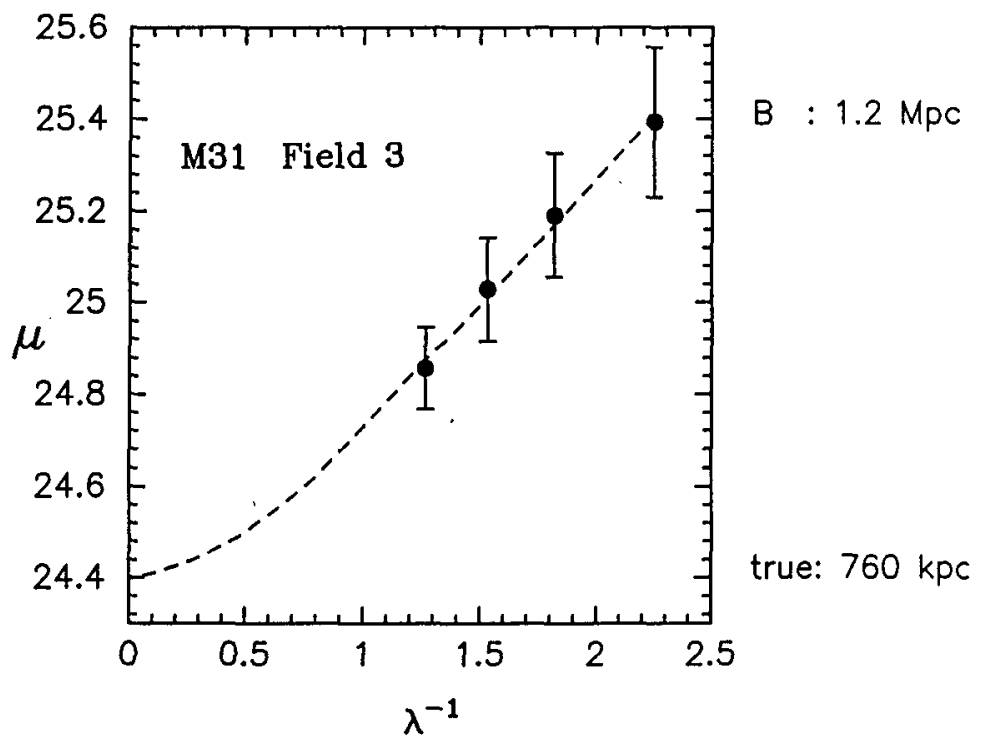

Figure 1. Apparent $B V R I$ distance moduli versus inverse wavelength for Cepheids in M31, Baade and Swope's Field III.

To summarize, the apparent excellent agreement between the Sandage and Tammann (1974) and the Madore and Freedman (1991) calibrations is misleading. The ST 1974 calibration is based on apparent $B$ band distance moduli, uncorrected for the effects of internal interstellar extinction; it is based on photographic photometry demonstrated as having significant scale errors, and finally, the MF 1991 calibration has a different zero point. The remarkable aspect of Tammann's Table 1 may not be the good agreement of those two particular calibrations, but rather that despite the fact that the distances to some calibrators individually changed by almost a factor of two in the interval between 1974 and 1992, the value of the Hubble constant derived by him and his collaborators showed very little change within the same period of time.

\section{A Comparison of Cepheid Distances with Other Methods}

Table 2 presents a comparison of recent distance modulus determinations to the galaxies M31, M33, the LMC and IC 1613. Included are distances obtained using new CCD or infrared photometry for Cepheids, RR Lyraes, and the tip of the red giant branch (TRGB). And finally the Type II supernova expansion parallax measurement of the distance to the LMC is shown for comparison. The RR Lyrae distances listed in Table 2 are based on assuming $M_{V}(R R)=+0.77$ mag. 
Table 2. Recent Distance Determinations to M31, M33, LMC and IC 1613

$\begin{array}{ccccc}\text { Method } & \text { M31 } & \text { M33 } & \text { LMC } & \text { IC } 1613 \\ & & & & \\ \text { Cepheids } & 24.44^{1} & 24.63^{2} & 18.50^{3} & 24.42^{4} \\ & & & 18.52^{5} & \\ \text { RR Lyraes } & 24.33^{6} & 24.45^{7} & 18.22^{8} & 24.10^{9} \\ \text { Giant Branch } & 24.40^{10} & 24.8^{11} & & 24.21^{12} \\ & & & & 24.27^{13} \\ \text { SN II } & & & 18.50^{14} & \end{array}$

References to Table 2: 1. Freedman and Madore (1990); 2. Freedman, Wilson and Madore (1990); 3. Welch et al. 1987; 4. Freedman (1988a); 5. Feast (1988); 6. Pritchet and van den Bergh (1987); 7. Pritchet (1988); 8. Walker (1992); 9. Saha et al. (1992); 10. Mould and Kristian (1986); 11. Mould and Kristian (1986); 12. Freedman (1988b); 13. Lee, Freedman and Madore (1992) 14. Panagia et al. (1991)

The first point to note about Table 2 is that the distances to individual galaxies based on both Population I and II indicators have converged to (full range) differences of less than $0.3 \mathrm{mag}$ (or $15 \%$ in distance). The Pop I and II distance scales have been completely independently calibrated, the former based on main sequence fitting of Galactic clusters, and the latter based on statistical parallax and Baade-Wesselink analyses of field RR Lyraes. Furthermore, as discussed below, the calibration of the RR Lyraes is still a matter of some controversy, and other published calibrations in fact result in an improvement of the agreement between the Cepheid and RR Lyrae distance scales. Nevertheless, despite remaining subtle calibration uncertainties, the good agreement of the distances to nearby galaxies indicates that the remaining controversy over the Hubble constant is due almost entirely to the uncertainties in the secondary distance methods.

Furthermore, as illustrated graphically in Figure 2, the good agreement amongst the various methods also suggests that there are no large systematic errors in the distance scale still lurking which might be due to the effects of metallicity (say). In Figure 2, differences in distance moduli are plotted versus estimates of $[\mathrm{Fe} / \mathrm{H}]$ determined for the halo populations in each of these galaxies. Shown are the differences in the Cepheid minus RR Lyrae, Cepheid minus tip of the red giant branch (TRGB), and RR Lyrae minus TRGB distances. Open circles indicate distances based on the assumption that $M_{V}(R R)=0.77 \mathrm{mag}$, whereas the dots represent distances based on a recent calibration by da Costa and Armandroff (1990) where $M_{V}(R R)=0.17$ $[\mathrm{Fe} / \mathrm{H}]+0.82$.

The second point to note about Table 2 is that although the Cepheid and RR Lyrae distance estimates agree to within their stated errors, the differences are systematic. The quoted uncertainties in the RR Lyrae moduli include a component to reflect the current uncertainty in the RR Lyrae zero point. In both the LMC and IC 1613 the zero point difference amounts to $+0.3 \mathrm{mag}$ (in the sense that the RR Lyrae distances 
are smaller than the Cepheid distances). Unfortunately the RR Lyrae distance for M33 is a preliminary estimate only, and may not provide additional information concerning the reality of such a systematic difference. Finally, in M31 the discrepancy is small (but the sense of the discrepancy is the same). Further work is clearly needed to establish the magnitude and/or reality of this effect.

As discussed recently by many authors (e.g. Sandage and Cacciari 1990; Carney et al. 1992; Walker 1992), the dependence of $M_{V}(R R)$ on metal abundance is not well-established. Extragalactic determinations of distances based on RR Lyraes have adopted the standard $M_{V}(R R)=0.77 \mathrm{mag}$, a value based on statistical parallax measurements of a sample of Galactic RR Lyraes, in which there are few metal-poor stars. Unfortunately, most of the extragalactic RR Lyrae stars known have been found in the metal-poor halos of the galaxies.
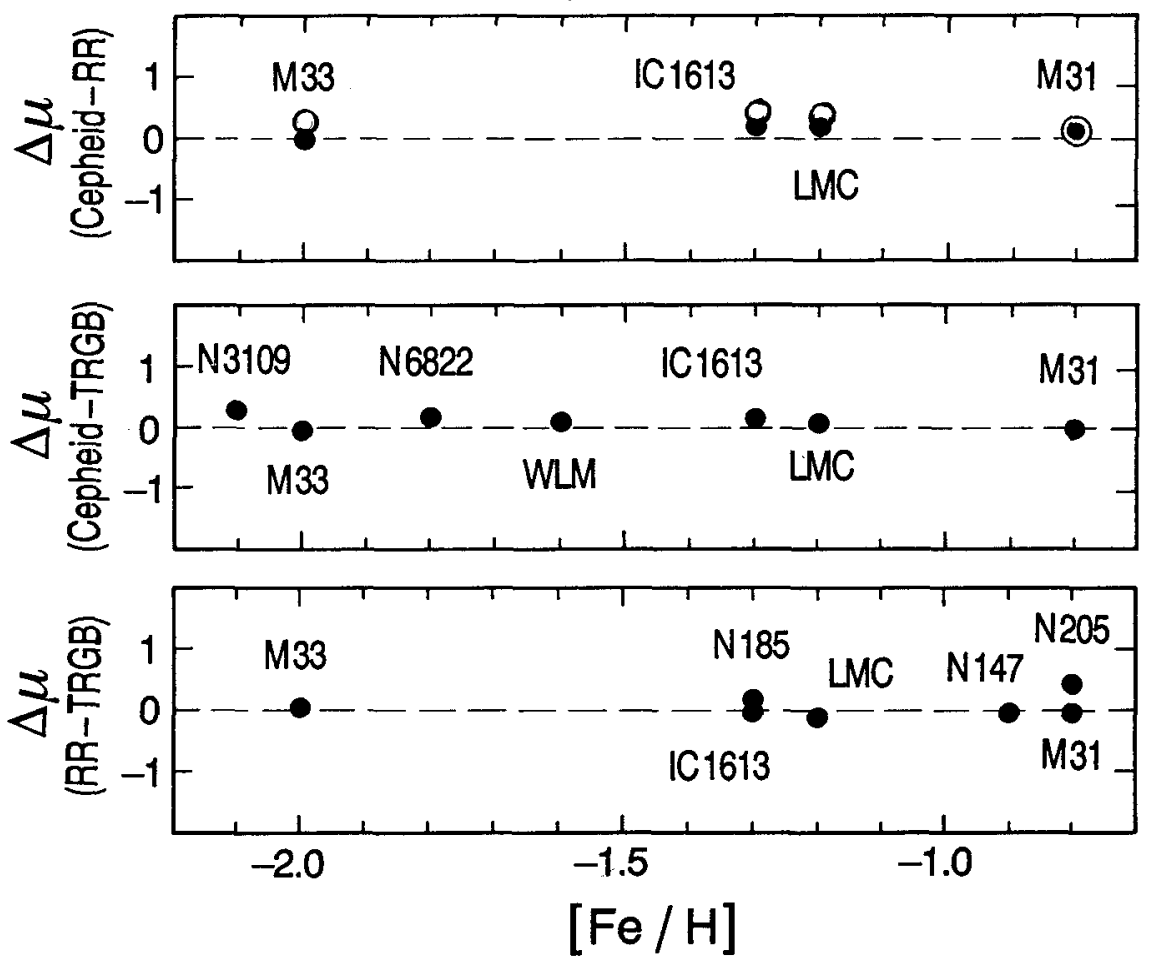

Figure 2. Differences in distance moduli as a function of halo $[\mathrm{Fe} / \mathrm{H}]$. (1) (Cepheid - RR Lyrae); (2) (Cepheid - TRGB); (3) (RR Lyrae - TRGB).

As seen in Figure 2, agreement between the Cepheid and RR Lyrae distance scales is improved if, for example, the recent calibration by $\mathrm{Da}$ Costa and Armandroff (1990) is adopted. The slope of the latter calibration agrees well with recent BaadeWesselink studies of Galactic RR Lyrae stars (Carney et al.), but the zero point differs by $0.2 \mathrm{mag}$, in better agreement with the Cepheid distances. The disadvantage of 
the Da Costa and Armandroff calibration (which is based on the theoretical models of Lee, Demarque and Zinn 1990), is that it carries with it associated uncertainties in modelling red giant branch evolution; for example mass loss on the red giant branch is not well-understood; the calibration is also dependent on horizontal branch evolution, and is very sensitive to the adopted helium abundance.

Alternatively, is the Cepheid distance scale in error? For example, is the Cepheid distance modulus to the LMC correct? Or are there systematic effects in the Cepheid distance scale due to metallicity? The excellent agreement between the Cepheid and SN 1987A expansion parallax estimates listed in Table 2 suggests that the LMC Cepheid distance is not seriously in error. As discussed by Saha et al. (1992), a systematic error in the Cepheid moduli due to metallicity appears unlikely since a theoretically-predicted metallicity-dependence of the Cepheid distance scale would actually increase, rather than resolve, the observed discrepancy.

It is worth noting however that the implications of such a discrepancy, if confirmed, may be profound. In particular, if the Cepheid distances are correct, (the point of view adopted by Walker 1992) it would imply that the absolute magnitudes of $R R$ Lyraes are brighter than currently believed. And an adjustment to the RR Lyrae distance scale zero point of $0.3 \mathrm{mag}$ would result in a decrease in the ages of globular clusters by about $30 \%$. It is interesting to note that while the distances to nearby galaxies now appear to have converged to a level where they have a small impact on uncertainty in the Hubble constant, subtle differences of only a few tenths of a magnitude may still have an impact on cosmology, through the ages determined from stellar evolution. Although the ages of globular clusters are widely regarded as theoretically-determined quantities, in the process of determining ages, it is still necessary to interface theory with observation and transform the apparent magnitudes of globular cluster stars to bolometric luminosities (via an accurate distance scale). It is also worth recalling in this context that this dependence of ages on distance is true for all methods of determining absolute (rather than simply relative) globular cluster ages (e.g., Renzini 1991).

\section{Using HST for the Discovery of Cepheids}

The discovery of Cepheids from space offers a powerful advantage over groundbased searches, even beyond the vital improvement offered in potential resolution. That is, from space, one has the unprecedented luxury of specifying the timing of the observations in order to to minimize aliasing problems in the period determination and maximize phase coverage in the time-averaged magnitude determinations; furthermore, the spacing is not altered by weather conditions.

In December 1991, the first sequence of HST observations of two fields in the relatively nearby galaxy M81 were obtained as part of the Key Project on the Extragalactic Distance Scale. ${ }^{1}$ Our only constraint was that the roll angle of the spacecraft

\footnotetext{
${ }^{1}$ Based on observations with the NASA/ESA Hubble Space Telescope, obtained at the Space Telescope Science Institute, which is operated by AURA under contract NAS5-26555.
} 
had to be kept within a narrow range, (consistent with properly illuminating the solar panels yet still allowing us to point back to the same position unrotated throughout the observing sequence). Consequently we were restricted to a 50-day initial observing interval. A power-law spacing algorithm (Madore and Freedman 1993) was developed to space the observations in such a way as to provide almost uniform phase coverage for Cepheids with periods in the range 5 to 50 days. To date 12 epochs of data at $V$ and 4 epochs at $I$ have been obtained for the two fields. Six additional observations have been scheduled to allow us to improve the periods (particularly important for the longest-period Cepheids, for which few, if any, complete cycles have been observed within the 50 -day window).

To date the results of the observing strategy and variable star search techniques have been very encouraging. In total, about two dozen candidate Cepheids have been discovered thus far in the two fields, increasing by an order of magnitude the number of Cepheids with known periods in this galaxy. The previously known Cepheid V30 was recovered and its 30 -day period confirmed. The data were reduced using a new version of DAOPHOT (named ALLFRAME) developed by another member of the Key Project Team, Peter Stetson. ALLFRAME was designed explicitly for the purpose of reducing a series of frames all covering the same field, and incorporates an empirically-generated PSF that varies with position on the chip. Figure 3 presents a light curve of one of the new Cepheid candidates. The light curve is of excellent quality, indicating that, with care, HST can produce photometry of very high precision. Moreover, with the success of our M81 effort and the similar work on IC 4182 (reported by Saha in this volume) HST is now accomplishing one of the tasks it was originally designed for and intended to do (namely the discovery of Cepheids in external galaxies, and the determination of the Hubble constant).

$$
\text { Star } 75 \quad \text { Period }=29^{d}
$$

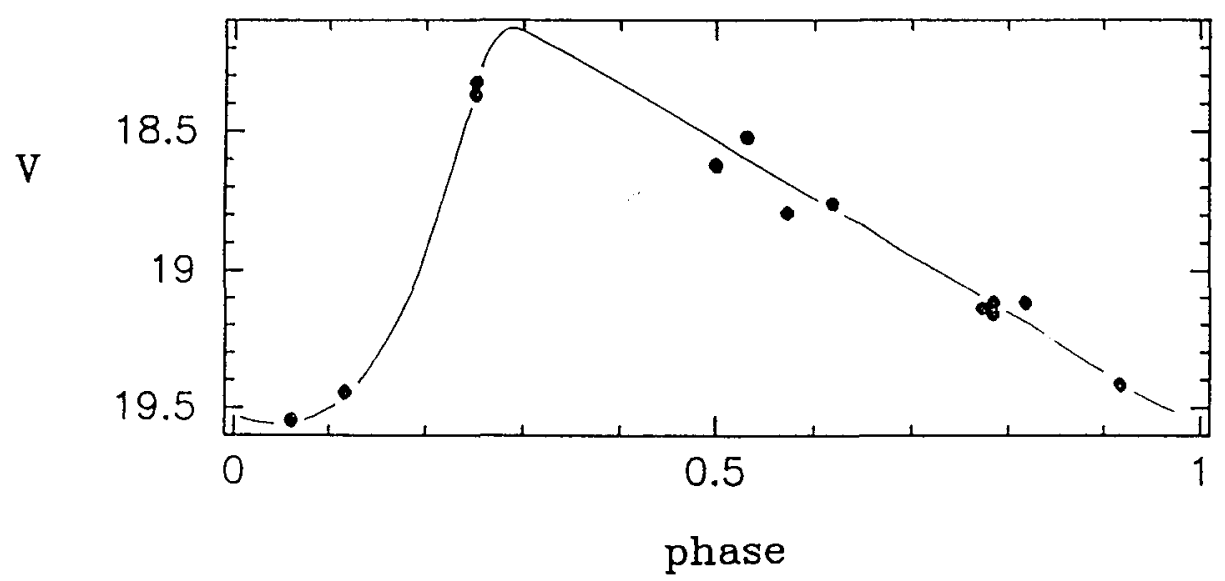

Figure 3. Light curve for one of the new HST Cepheid candidates in M81. 
Obviously, with the HST discovery of Cepheids for a single galaxy in our program, it is premature to derive a value of the Hubble constant (however, see Sandage et al. 1992).

Encouraged by the quality of the photometry for the M81 Cepheids the HST Key Project Team is intending to look for Cepheids in 2 fields in M101 during upcoming cycles. An inner and an outer field in M101 will provide us with an opportunity to undertake a further test for the metallicity effects in the Cepheid distance scale, as done previously for Cepheids in M31 (Freedman and Madore 1991). When the HST optics have been corrected we intend to then pursue our long-term program of measuring the distances to a statistically significant sample of nearby spirals.

It is a pleasure to acknowledge the efforts of the additional Key Project Team members: Sandy Faber, Holland Ford, Jim Gunn, John Hoessel, John Huchra, Rob Kennicutt, Garth Illingworth, Jeremy Mould, and Peter Stetson, and especially Shaun Hughes and Myung Gyoon Lee. The work on the red giant branch distance scale has been done in collaboration with Myung Gyoon Lee. WLF acknowledges continual stimulating discussions with Allan Sandage, particularly in the Santa Barbara St. library. We have profitted greatly from the use of the NASA/IPAC Extragalactic Database (NED). Support for this work was provided in part by NSF grant AST-9116496 and by NASA through grant numbers GO-227.04-87A. BFM is supported in part by JPL Caltech, under the sponsorship of NASA's Office of Space Science and Applications.

\section{References:}

Carney, B., Storm, J. \& Jones, R. V., 1992, ApJ, 386, 663

Da Costa, G.S., Armandroff, T.E., 1990, AJ 103, 1151

Feast, M. 1988, in "The Extragalactic Distance Scale", ASP Conference Series Vol. 4, eds. S. van den Bergh \& C. Pritchet (Provo, Brigham Young University Press), p. 9

Feast, M., \& Walker, A. R. 1987 ARA\&A, 25, 345

Fernie, J. D. 1969, PASP, 81, 707

Freedman, W. L. 1986, in Galaxy Distances and Deviations from Universal Expansion, eds. B. F. Madore \& R. B. Tully, (Dordrecht: Reidel), p. 21

Freedman, W. L. 1988a, in "The Extragalactic Distance Scale", ASP Conference Series Vol. 4, eds. S. van den Bergh \& C. Pritchet (Provo, Brigham Young University Press), p. 24

Freedman, W. L. 1988b, ApJ, 326, 691

Freedman, W. L., \& Madore, B. F. 1990, ApJ, 365, 186

Freedman, W. L., Wilson, C., \& Madore, B. F. 1991, ApJ, 372, 455

Jacoby, G. H. et al., 1992, PASP, 104, 599

Lee, Y.-W., Demarque, P., and Zinn, R. 1990, ApJ, 350,155

Lee, M. G., Freedman, W. L., \& Madore, B. F. 1993, in preparation 
Madore, B. F. 1986, in "Galaxy Distances and Deviations from Universal Expansion”, eds. B. F. Madore \& R. B. Tully (Dordrecht, Reidel), p. 29

Madore, B. F. \& Freedman, W. L. 1991, PASP, 103, 933

Madore, B. F. \& Freedman, W. L. 1993, in preparation

Mould, J. \& Kristian, J. 1986, AJ, 305, 591

Panagia, N., Gilmozzi, R. Macchetto, F., Adorf, H.-M., \& Kirshner, R. P. 1991, ApJ, 380, L23

Pritchet, C. J. 1988, in "The Extragalactic Distance Scale", ASP Conference Series Vol. 4, eds. S. van den Bergh \& C. Pritchet (Provo, Brigham Young University Press), p. 59

Renzini, A. 1992, in “Observational Tests of Inflation", eds. T. Banday \& T. Shanks, (Dordrecht: Reidel), in press

Saha, A., Freedman, W. L., Hoessel, J G., \& Mossman, A. E., AJ, 104, 1072

Sandage, A. R. 1983, AJ, 88, 1108

Sandage, A. R. 1984, AJ, 89, 621

Sandage, A. R. 1988, ApJ, 331, 605

Sandage, A. R., \& Cacciari, C. 1990, ApJ, 350, 645

Sandage, A. R., \& Carlson, G. 1983a, ApJ, 267, L25

Sandage, A. R., \& Carlson, G. 1983b, ApJ, 258, 439

Sandage, A. R., Saha, A., Tammann, G. A., Panagia, N., \& Macchetto, F. 1992, ApJ, in press

Sandage, A. R., \& Tammann, G. A. 1974, ApJ, 190, 525

Tammann, G. A. 1992, The Cosmic Expansion and Deviations from It, to appear in Physica Scripta

Tammann, G. A., \& Sandage, A. R. 1968, ApJ, 151, 825

Walker, A. R. 1992, ApJ, 390, L81

Welch, D. L., McLaren, R., Madore, B. F., \& McAlary, C. W. 1987, ApJ, 321, 162 